\author{
M. SCHARTE ${ }^{1}$ \\ R. PORATH ${ }^{1}$ \\ T. OHMS ${ }^{1}$ \\ M. AESCHLIMANN ${ }^{1}$ \\ J.R. KRENN ${ }^{2}$ \\ H. DITLBACHER ${ }^{2}$ \\ F.R. AUSSENEGG ${ }^{2}$ \\ A. LIEBSCH $^{3, \infty}$
}

\section{Do Mie plasmons have a longer lifetime on resonance than off resonance?}

\author{
${ }^{1}$ Department of Physics, University of Kaiserslautern, 67663 Kaiserslautern, Germany \\ ${ }^{2}$ Institute for Experimental Physics, Karl-Franzens University, 8010 Graz, Austria \\ ${ }^{3}$ Institut für Festkörperforschung, Forschungszentrum Jülich, 52425 Jülich, Germany
}

\section{Received: 28 August 2001}

Published online: 15 October 2001 • C Springer-Verlag 2001

ABSTRACT The dynamics of laser-excited electrons in elliptically shaped silver nano-particles is studied using time-resolved two-photon photo-emission. By changing the polarization of the laser pulse at a fixed frequency, a different dynamics is observed near and far from resonance. To analyze these data, the effect of radiation damping on Mie plasmons of Ag nanoparticles is studied as a function of laser frequency and polarization. It is shown that the off-resonance lifetime can be shorter or longer than the lifetime at resonance, depending on the incidence conditions of the laser light and on the depolarization factors characterizing the optical response of the nano-particles.

PACS 36.40.Gk; 61.46.+w; 78.47.+p

\section{1}

\section{Introduction}

The optical properties of nano-structured systems have been extensively investigated in order to elucidate their fundamental processes and to examine possible technological applications $[1,2]$. In metal nano-particles, collective electronic oscillations - the so-called Mie plasmons - can be excited by light and are therefore detectable as a pronounced optical resonance in the visible or UV parts of the spectrum. The collective oscillation can be interpreted as a displacement of the center of mass of all electrons in the particle against the positively charged background of the atomic cores. This resonance dominates the linear and nonlinear response of the materials. Considerable interest has been focused on silver nanoparticles as they exhibit a particularly strong size-dependent optical resonance in the visible spectral range $(1.8-3 \mathrm{eV})$, i.e., at energies below the interband transition threshold $(\approx 4 \mathrm{eV})$. Consequently, the absorption cross-section in the visible region is dominated by Drude damping [3]. The contribution due to interband transitions is negligible.

In recent years several line width measurements and timeresolved second harmonic generation autocorrelation measurements on metallic nano-particles have been published, reporting a dephasing time of the Mie-plasmon excitation on the order of 4-20 fs [4-8]. Much less is known, however,

Fax: +49-2461/612-850, E-mail: a.liebsch@fz-juelich.de about the microscopic mechanism of the investigated damping rate. The excited electronic system in large nano-particles $(>50 \mathrm{~nm}$ ) can exhibit additional dominant microscopic relaxation channels that are not important in bulk material, such as radiation damping. Compared with Drude damping the radiation damping is explicitly frequency dependent. The question arises whether the same microscopic damping processes due to photons, phonons, impurities, surface scattering and electron-electron interactions are involved when the photoexcitation is close to the Mie plasma resonance or far from it.

The present work focuses on time-dependent studies of elliptically shaped silver nano-particles with dimensions of $80 \mathrm{~nm} \times 40 \mathrm{~nm}$. In particular, autocorrelation measurements with femtosecond laser pulses were performed by two-photon photoemission (TR-2PPE) in order to determine the dynamics of the optically excited electronic system. The two-dimensional array of nearly identical, parallel-oriented particles are deposited lithographically onto a transparent indium tin oxide (ITO) substrate, which itself lies on a glass plate. The size of the particles was chosen such that quantum confinement effects could be neglected. Elliptically shaped metal nano-particles show two different plasmon resonance frequencies for light polarized parallel to the short and long axes, respectively. Choosing the laser wavelength at one of these two resonances makes it possible to distinguish between excitation near and far from resonance by simply rotating the polarization of the laser pulse. The measurements were performed in the low perturbation regime in order to avoid space charge effects and deformation of the metallic nano-particles.

\section{2}

\section{Experiment}

The experimental method used to investigate the dynamics of the optically induced electronic excitations was time-resolved two-photon photoemission. This pump-probe technique enables a direct measurement of the dynamical properties in the time domain with a resolution of a few femtoseconds [9]. An ultrashort (pump) laser pulse excites electrons of the valence band to unoccupied states with energies between the Fermi and vacuum level. A second (probe) laser pulse measures the transient population of these intermediate states as a function of the temporal delay with respect to the pump pulse. The measured pump-probe signal (autocorrelation curve, see below) contains an exponentially decaying 


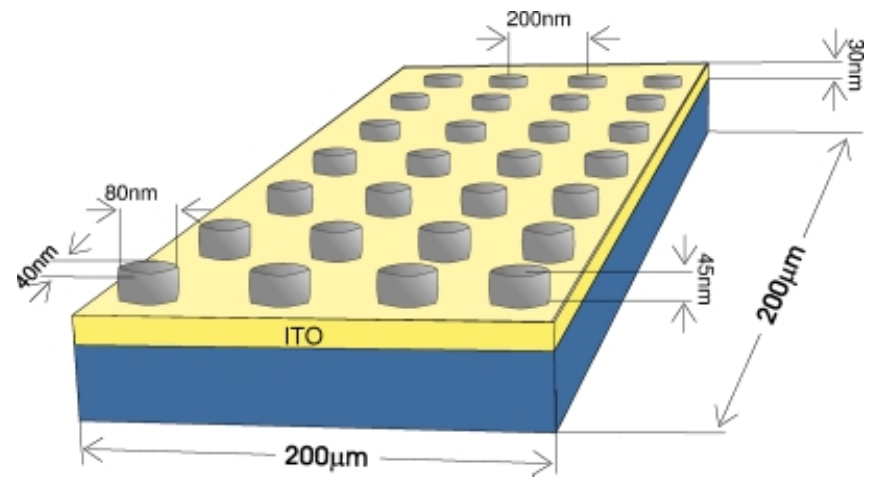

FIGURE 1 Array of Ag nano-particles deposited on the ITO substrate

contribution which is, within a first-order approximation, directly correlated to the population decay time $T_{1}$ of the intermediate excitation state.

The experiments were performed with a femtosecond Kerr lens mode-locked Ti:sapphire laser, pumped by about $10 \mathrm{~W}$ from a cw-operated diode pumped solid-state laser. The system delivered transform-limited and $\operatorname{sech}^{2}$ temporally shaped pulses with up to $12 \mathrm{~nJ} /$ pulse and a duration of $40 \mathrm{fs}$ at a repetition rate of $82 \mathrm{MHz}$ and a wavelength of $830 \mathrm{~nm}$. The linearly polarized output of the Ti:sapphire laser was frequencydoubled in a 0.2 -mm-thick beta barium borate (BBO) crystal in order to produce UV pulses at $\hbar \omega=2.99 \mathrm{eV}$, corresponding to $415 \mathrm{~nm}$. In a Mach-Zehnder interferometer the pulses were divided by a beamsplitter into equal intensity (pump and probe) pulses. One path was delayed with respect to the other by a computer-controlled delay stage. Both beams were recombined collinearly by a second beamsplitter, sent through an adjustable $\lambda / 2$-retardation plate and focused perpendicularly to the sample surface. The diameter of the laser spot on the sample was around $100 \mu \mathrm{m}$. It was possible to rotate the plane of polarization to any arbitrary angle by means of the $\lambda / 2$ plate. Nevertheless the light was always $s$-polarized with respect to the sample surface.

The sample was mounted in an ultra-high vacuum chamber held at a base pressure in the $10^{-10}$ mbar range. A bias voltage of $-4 \mathrm{~V}$ was applied to the sample to eliminate effects of any stray electric fields. The photo-emitted electrons were detected in a cylindrical sector analyzer (CSA). The entrance axis of the energy analyzer was $45^{\circ}$ with respect to the laser beam. In general, we used a pass energy of $4 \mathrm{eV}$, leading to a resolution of roughly $50 \mathrm{meV}$.

The long and short axes of the elliptically shaped silver nano-particles were $80 \mathrm{~nm}$ and $40 \mathrm{~nm}$, respectively. Their height was $45 \mathrm{~nm}$. Figure 1 illustrates the geometry, size and the distances of the particles in the array. In the following the long half-axis of the elliptic particle is called $a$ and the short half-axis is called $b$. The whole quadratic array containing the particles had a lateral length of $200 \mu \mathrm{m}$. The substrate was a 30-nm-thick layer of ITO which itself was placed on a quartz glass plate. ITO was used because on the one hand it is electrically conductive, preventing charging effects, but on the other hand the amount of photo-emitted electrons coming from its surface is negligible. An electron-beam-lithography technique was used for a controlled design of the metallic nano-particles [10]. As particle shape and interparticle dis-

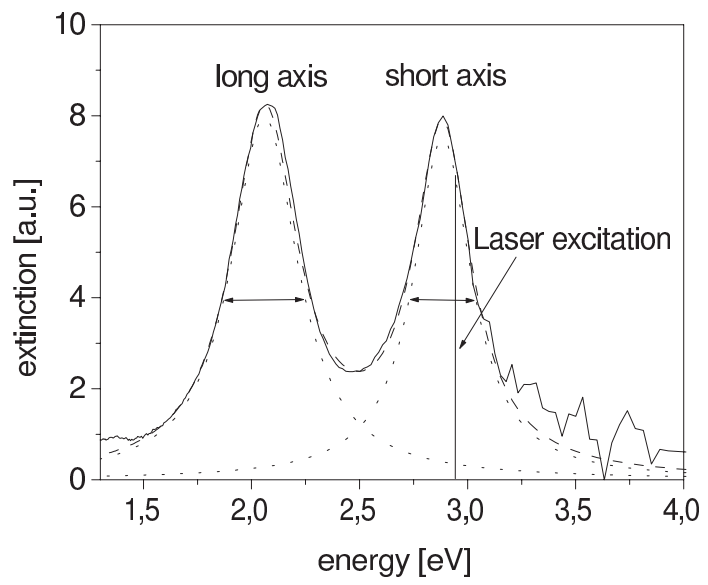

FIGURE 2 Measured extinction spectrum of the nano-particle array. The low- and high-frequency peaks correspond to the Mie plasmons excited along the long and short axes of the elliptical Ag particles, respectively

tance can be varied independently, this method allows the optical properties of individual particles to be tailored. Thus the resonance frequency and the strength of particle interaction can be tuned independently. Thereby the optical extinction maximum can be tuned to the desired wavelength (in this case to the illuminating laser wavelength of $415 \mathrm{~nm}$ ). The nano-particle has two different resonances, as is visible in the extinction spectrum (Fig. 2): $\hbar \omega_{a}=2.1 \mathrm{eV}$ and $\hbar \omega_{b}=2.9 \mathrm{eV}$. Both peaks have a FWHM of about $0.4 \mathrm{eV}$.

Figure 3 shows a typical autocorrelation trace as obtained from a TR-2PPE measurement at an intermediate state energy, $E-E_{\mathrm{F}}=1.8 \mathrm{eV}$ [11]. A $\operatorname{sech}^{2}$ curve is fitted to the experimental data in order to obtain the FWHM of the trace. In Fig. 4 the FWHM derived from the autocorrelation traces are plotted as a function of the rotation of the state of polarization. For comparison the behavior of the polycrystalline tantalum is also shown. The FWHM for tantalum is not affected by turning the polarization angle of the incoming light. Therefore, any effect caused by an increase in the dispersion due to rotation of the $\lambda / 2$ plate can be excluded. For the $\mathrm{Ag}$ nano-particles, however, a rotation of $90^{\circ}$ reduces the FWHM from almost $75 \mathrm{fs}$ in the $b$-direction (short-axis mode, resonant plasmon excitation) to $69 \mathrm{fs}$ in the $a$-direction (long-axis

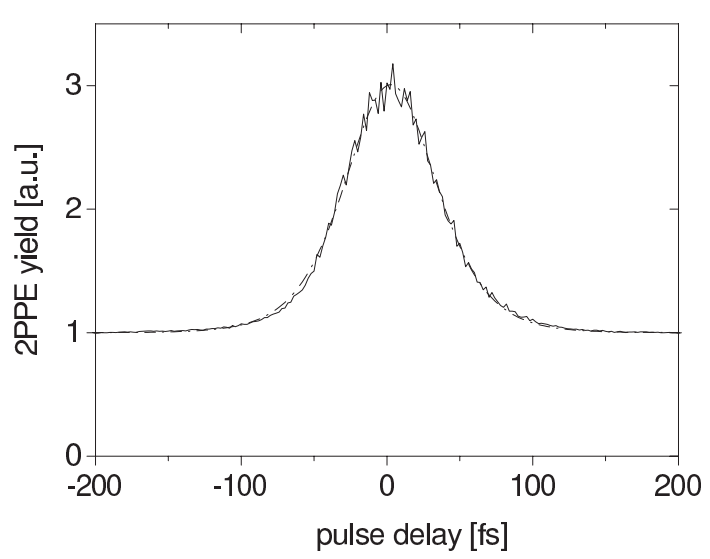

FIGURE 3 Typical autocorrelation measurement of time-resolved twophoton photo-emission yield as a function of delay time 


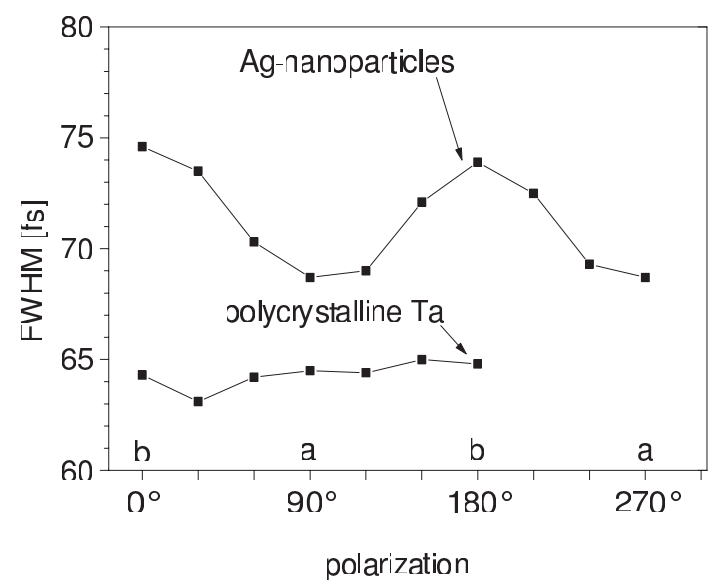

FIGURE 4 Full-width at half-maximum of the autocorrelation of the $\mathrm{Ag}$ particle array and polycrystalline Ta as a function of laser polarization in the plane of the surface. $a$ : excitation along long axis; $b$ : excitation along short axis. The laser frequency is $2.99 \mathrm{eV}$

mode, off-resonance plasmon excitation). Further rotation restores the long lifetime of the plasmon excitation case. One should be aware that these FWHM values still include the autocorrelation of the laser pulse width. The autocorrelation traces of tantalum were measured at an intermediate state energy, $E-E_{\mathrm{F}}=2.8 \mathrm{eV}$. In this energy range, the lifetime of excited electrons is less than $1 \mathrm{fs}$ [12], and hence the traces obtained for tantalum represent the pure laser autocorrelation curve. Therefore, any increase in the FWHM in the autocorrelation measurement for Ag nano-particles compared to the Ta values must be caused by a lifetime effect of the optically excited electron system.

\section{Theory}

Let us consider an isolated spheroidal metal particle of volume $V$ subject to an external electromagnetic field $E_{\text {ext }}$ of frequency $\omega$ (wavelength $\lambda$ ). The bulk dielectric function of the particle is $\varepsilon(\omega)=\varepsilon_{1}(\omega)+\mathrm{i} \varepsilon_{2}(\omega)$. Assuming the incident field to be oriented along one of the principal axes, the total induced polarization may be written as follows $(i=$ $x, y, z)[13,14]$ :

$P_{i}(\omega)=\chi_{i}(\omega)\left[E_{\mathrm{ext}}(\omega)+E_{\mathrm{rad}}(\omega)\right]$,

where the diagonal elements of the susceptibility tensor are given by

$\chi_{i}(\omega)=\frac{1}{4 \pi} \frac{\varepsilon(\omega)-1}{1+[\varepsilon(\omega)-1] A_{i}}$.

For ideal spheroids, the depolarization factors $A_{i}$ satisfy the sum rule $A_{1}+A_{2}+A_{3}=1$; in the case of a sphere, $A_{i}=1 / 3$. The second term in (1) accounts for losses due to radiation and is related to the polarization via

$E_{\mathrm{rad}}(\omega)=4 \pi \mathrm{i} B(\omega) P_{i}(\omega)$,

where the coefficient $B(\omega)$ is defined as

$B(\omega)=\frac{4 \pi^{2}}{3} \frac{V}{\lambda^{3}}$.
This relationship may be derived by equating the work done by $E_{\mathrm{rad}}$ on the dipole with the radiated energy [15]. Eliminating $E_{\mathrm{rad}}$ from (1), the polarization takes the form

$P_{i}(\omega)=\frac{\chi_{i}(\omega)}{1-4 \pi \mathrm{i} B(\omega) \chi_{i}(\omega)} E_{\text {ext }}(\omega)$.

To find the electric field inside the particle, we use

$\varepsilon(\omega) E_{\mathrm{int}}(\omega)=E_{\mathrm{int}}(\omega)+4 \pi P_{i}(\omega)$,

which yields $E_{\text {int }}(\omega)=f_{i}(\omega) E_{\text {ext }}(\omega)$, where the dynamical field enhancement factor $f_{i}(\omega)$ is given by

$f_{i}(\omega)=\frac{1}{1+[\varepsilon(\omega)-1]\left[A_{i}-\mathrm{i} B(\omega)\right]}$.

In the following we focus on Ag nano-particles, whose bulk optical properties are well represented by writing the dielectric function as

$\varepsilon(\omega)=1-\frac{\omega_{p}^{2}}{\omega(\omega+\mathrm{i} \gamma)}+\left[\varepsilon_{d}(\omega)-1\right]$.

The first term is the Drude function characterizing the nearlyfree $5 s$ electrons, where $\omega_{\mathrm{p}}=9.2 \mathrm{eV}$ is the plasma frequency and $\gamma$ the weakly frequency-dependent damping parameter. The second term accounts for the optical response of the occupied $4 d$ bands.

Although (7) can be used to analyze the observed extinction spectrum, it is not obvious how to extract from the shape of $f_{i}(\omega)$ the inverse lifetime $\Gamma_{i}(\omega)$ of the plasma modes, in particular, its variation with frequency. To derive an expression for $\Gamma_{i}(\omega)$, we rewrite the enhancement factor as follows: Since $\gamma \ll \omega$ and $\varepsilon_{d}(\omega)$ is real in the region of interest, the resonance denominator of $f_{i}$ can be approximated as

$$
\begin{aligned}
f_{i}^{-1}(\omega)=1 & +\left[\left(\varepsilon_{1}-1\right) A_{i}+\varepsilon_{2} B\right]+\mathrm{i}\left[\varepsilon_{2} A_{i}-\left(\varepsilon_{1}-1\right) B\right] \\
\approx 1 & +\left[\left(\varepsilon_{d}-1-\frac{\omega_{\mathrm{p}}^{2}}{\omega^{2}}\right) A_{i}+\varepsilon_{2} B\right] \\
& +\mathrm{i}\left[\frac{\omega_{\mathrm{p}}^{2}}{\omega^{2}} \frac{A_{i}}{\omega}\left(\gamma+\omega \frac{B}{A_{i}}\right)-\left(\varepsilon_{d}-1\right) B\right] .
\end{aligned}
$$

The resonance frequency is determined by the vanishing real part of $f_{i}^{-1}$ and is given by

$\omega_{i} \approx \frac{\omega_{\mathrm{p}}}{\sqrt{\varepsilon_{d}-1+A_{i}^{-1}}}$,

where we have neglected the small shift due to $\varepsilon_{2} B$. Thus, the frequency of the Mie plasmon $\omega_{i}$ is determined to good accuracy by the geometrical depolarization factor $A_{i}$. Radiation causes only a weak redshift.

As can be seen from the imaginary part of $f_{i}^{-1}$, the overall broadening due to Drude damping and radiation losses is given by the function

$\Gamma_{i}(\omega) \approx \gamma+\omega B(\omega) / A_{i}$.

The small correction associated with the term $\left(\varepsilon_{d}-1\right) B$ is omitted. For the particle sizes considered below, the damping due to radiation is rather large and dominates the Drude 
damping. Moreover, since $\omega B(\omega) \sim \omega^{4}$, radiation damping is strongly frequency dependent and leads to a skewing of the plasmon peak. In particular, this implies that the damping near the maximum of the plasmon resonance is not representative of the damping far from resonance. Below the resonance, radiation damping is weaker than at resonance, while above the resonance, it becomes rapidly stronger.

Another important result is that the radiation reaction field defined in (3) leads to different damping along the axes of the ellipsoid. Since the polarization along these axes is governed by the factors $A_{i}$, the induced damping is also determined by these coefficients. The following simple rule can be derived from the above expressions: A long ellipsoidal axis implies a low Mie resonance frequency, $\omega_{i}$, a small depolarization factor, $A_{i}$, and a strong radiation damping, $\omega B(\omega) / A_{i}$. Conversely, a short axis gives a higher Mie frequency, a larger depolarization factor, and weaker radiation damping. These trends are consistent with the expectation that for increasing ratio $R_{i} / \lambda\left(R_{i}\right.$ is the radius along axis $i$ ), the influence of radiation damping should also increase.

In the case of ordered particle arrays deposited on a dielectric substrate, dipole and multipole interactions between particles and interactions with the substrate induce shifts of the Mie plasma frequencies. Furthermore, deviations from the ideal ellipsoidal shape cause mixing between the principal modes. Since these effects are difficult to describe quantitatively, we use the depolarization factors $A_{i}$ as fitting parameters in order to reproduce the experimentally observed extinction spectrum for normal incidence. In this configuration only, the modes parallel to the surface plane are detected in which the particles have a nearly perfect elliptical shape, with semi-major axis $a$ and semi-minor axis $b$. The corresponding depolarization factors are denoted by $A_{a}$ and $A_{b}$, respectively. For light polarization along these axes, mixing between the associated plasma modes should be small.

Figure 5 shows the imaginary part of the field enhancement factor $f_{i}(\omega)$ for two effective depolarizations, $A_{a}=$ 0.061 for the long axis (radius $a=40 \mathrm{~nm}$ ) and $A_{b}=0.145$ for the short axis (radius $b=20 \mathrm{~nm}$ ). The Ag dielectric function

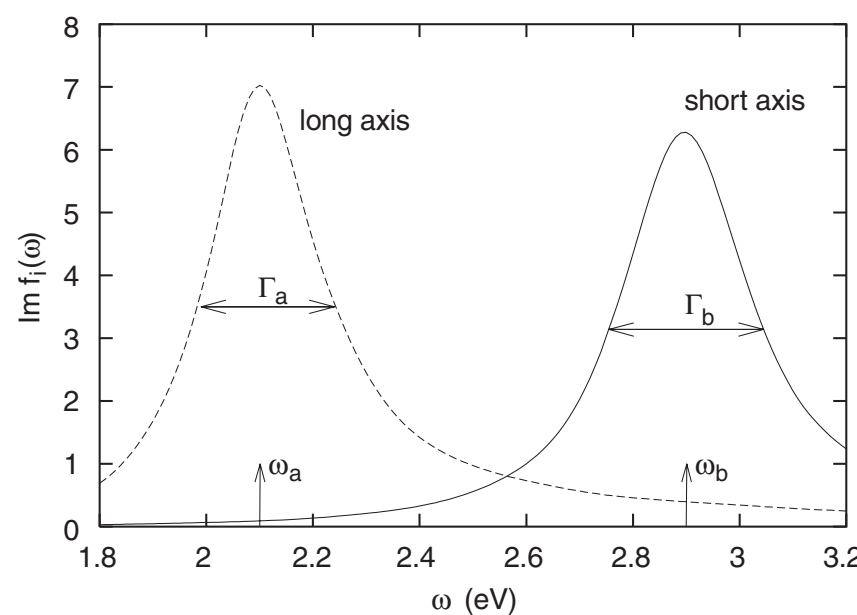

FIGURE 5 Imaginary part of the field enhancement factor $f_{i}(\omega)$ for depolarization factors $A_{b}=0.145$ representing the short ellipsoidal axis $b$ (solid curve) and $A_{a}=0.061$ representing the long axis $a$ (dashed curve) is taken from bulk optical data [16], and the particle volume is $V=\pi a b h$, with $h \approx 45 \mathrm{~nm}$ being the particle height. For comparison we note that the Mie plasmon of a spherical Ag particle in the absence of radiation losses lies at the frequency $\omega_{\mathrm{M}}=3.5 \mathrm{eV}$ and the width is $0.1 \mathrm{eV}$. As a result of the depolarization factors $A_{i}$ the Mie plasma frequencies in the present case are $\omega_{a}=2.1 \mathrm{eV}$ and $\omega_{b}=2.9 \mathrm{eV}$, respectively. These frequencies and the peak shapes agree well with the features observed in the extinction spectra shown in Fig. 2 from square arrays of Ag nano-particles deposited lithographically on a transparent ITO substrate, with dimensions as specified above [11].

At these plasma frequencies, the term $B(\omega)$ characterizing radiation damping is about 10 times smaller than the corresponding depolarization factors $A_{i}: B\left(\omega_{a}\right)=0.007$ and $B\left(\omega_{b}\right)=0.019$. Although the $B$ terms cause a negligible shift of the resonance peaks, they have an important effect on the lifetime broadening: Both plasmon features exhibit a width of about $0.4 \mathrm{eV}$, in good agreement with the data.

As pointed out above, radiation damping is strongly frequency dependent. At first, it is therefore surprising that both peaks exhibit about the same amount of radiation damping. However, as evident from (11), the radiation damping scales inversely with the polarization factor $A$. Thus, for the low (high) frequency Mie plasmon, both $\omega B(\omega)$ and $A$ are small (large), so that $\Gamma_{a}\left(\omega_{a}\right) \approx \Gamma_{b}\left(\omega_{b}\right)$.

An interesting question arises concerning the lifetime of the plasma oscillation in the nano-particle near resonance and off resonance. In small spherical Ag particles, this damping is given by the Drude parameter $\gamma$, which is weakly frequency dependent, since it stems primarily from impurity and phonon scattering as well as small contributions due to electronelectron interactions [3]. Surface scattering plays a role only at radii less than about $50 \AA[2,17]$. Thus, for particle radii of about $50-100 \AA$, the damping can be regarded as frequency independent; in particular, it has the same value near the resonance frequency as above or below the peak maximum. The physical reason is, of course, that below the interband onset near $4 \mathrm{eV}$ all electrons are subject to the same microscopic scattering processes, regardless of whether they oscillate at resonance or not. The plasma mode signifies a larger amplitude of oscillation but not the presence or absence of a particular microscopic scattering mechanism.

For larger nano-particles, this picture changes, since now the effective damping parameter is explicitly frequency dependent because of radiation losses, as indicated in (11). To illustrate this effect we compare in Fig. 6 the functions $\Gamma_{i}(\omega)$ for the long and short ellipsoidal axes with the bare Drude damping $\gamma$ as derived from bulk optical data. At any fixed laser frequency, the long-axis mode is seen to exhibit about twice as much radiation damping as the short-axis mode (the ratio is essentially given by $A_{b} / A_{a} \approx 2.4$ ). The radiation damping for both modes is significantly larger than the Drude damping.

Let us now consider the lifetime measurement discussed in the previous section, i.e., two-photon photo-emission at the pump laser frequency near the peak of the short-axis mode at $2.9 \mathrm{eV}$. For a light polarization along the short $b$ axis, we would expect to observe a damping given approximately by $\Gamma_{b}=\Gamma_{b}\left(\omega_{b}\right)$. If we now rotate the polarization direction to- 


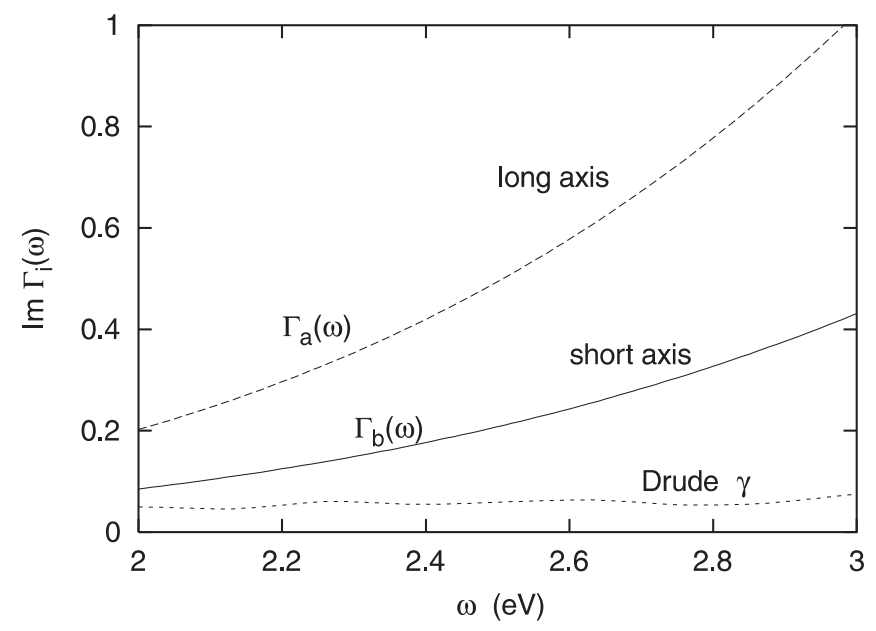

FIGURE 6 Plasmon broadening $\Gamma_{i}(\omega)$ as a function of frequency. Solid curve: damping of high-frequency mode $\Gamma_{b}(\omega)\left(\omega_{b}=2.9 \mathrm{eV}\right.$, short axis); dashed curve: damping of low-frequency mode $\Gamma_{a}(\omega)\left(\omega_{a}=2.1 \mathrm{eV}\right.$, long axis); dot-dashed curve: Drude damping $\gamma$ derived from bulk optical data

wards the long $a$ axis without changing the laser frequency, we excite the high-frequency tail of the $\omega_{a}$ plasma mode and expect to find the damping $\Gamma_{a}\left(\omega_{b}\right)$, which is about twice as large as $\Gamma_{b}$. Thus, in this particular configuration the collective mode $\omega_{b}$ detected on resonance has a much longer lifetime than the excitations in the high-frequency tail of the $\omega_{a}$ mode.

This trend agrees with the experimental observation using two-photon photo-emission at $3 \mathrm{eV}$ pump laser frequency. According to Fig. 4, the increase in the FWHM of the TR-2PPE autocorrelation curve is about a factor 2 smaller for the longaxis mode as compared to the short-axis mode, taking into account that the FWHM of the Ta measurements represents the FWHM of the laser autocorrelation curve.

It might be tempting to conclude from this analysis that on-resonance collective excitations in general have a longer lifetime than excitations far from resonance. This is not correct, however, since the exact opposite should be obtained if we perform the analogous experiment near the frequency $\omega_{a}$ of the long-axis mode. For a polarization along the $a$ axis, we observe this mode on resonance with a damping given by $\Gamma_{a}=\Gamma_{a}\left(\omega_{a}\right)$. (According to Fig. 6, this damping is accidentally similar to $\Gamma_{b}$.) If we rotate the polarization towards the short $b$ axis, while keeping the laser frequency fixed at $\omega_{a}$, we should observe a strong decrease in damping, since excitations of the nano-particle along the short axis are less subject to radiation losses. Thus, in this alternative configuration, the collective mode $\omega_{a}$ detected on resonance has a shorter lifetime than the excitations in the low-frequency tail of the $\omega_{b}$ mode. To test this theoretical prediction, it would be desirable to repeat the two-photon photo-emission measurement with a pump laser frequency of about $2.1 \mathrm{eV}$.

On the basis of the above theoretical analysis we can give the following tentative interpretation of the observed twophoton photo-emission spectra: The initial pump pulse excites an electron from an occupied state 1 to an intermediate state 2 . The effective field governing this excitation consists of a superposition of external field and induced field generated by the dynamical response of the electrons of the Ag particle. Ac- cordingly, the amplitude of this excitation process is greatly enhanced if the laser frequency coincides with the Mie resonance. Because of the strong intrinsic broadening of the Mie plasmon the induced contribution to the effective electromagnetic field exciting state 1 is coherent over rather short times, of the order of $0.6-6 \mathrm{fs}$, corresponding to $\Gamma \approx 0.1-1.0 \mathrm{eV}$ (see Fig. 6). Since the energy of the intermediate state is about $2 \mathrm{eV}$ above the Fermi energy, the excited electron scatters quasi-elastically via phonons and impurities and via inelastic electron-electron processes, giving a typical overall lifetime of about 6-10 fs, corresponding to an imaginary part of the $\mathrm{Ag}$ quasi-particle self-energy of about $0.06-0.1 \mathrm{eV}$ [18-21]. The probe beam excites the electron from the intermediate state to the final state 3 above the vacuum level such that it can be detected as an emitted electron. The probability of the second excitation process is also greatly enhanced if the probe laser is in resonance with the Mie plasma frequency. The probe pulse is subject to the same frequency-dependent damping mechanisms as the pump pulse. The measurements discussed in the present work suggest that time-resolved, two-photon photo-emission is sensitive not only to the decay of the intermediate electronic state but also to the intrinsic damping of the effective electromagnetic field causing the single-electron transitions.

\section{$4 \quad$ Summary}

The lifetime of Mie plasmons of Ag nano-particles was investigated using two-photon photo-emission spectroscopy. The different damping rates obtained under on- and off-resonance conditions were shown to be compatible with different magnitudes of radiation damping along the long and short axes of the elliptical particles. In general, at a given laser frequency, photo-excitation along the long axis implies stronger radiation damping than along the short axis. This result is consistent with the well-known fact that for spherical particles radiation losses become more pronounced with increasing radius. Moreover, this result holds regardless of whether the photo-excitation is close to the Mie plasma resonance or far from it. In fact, collective plasma oscillations within the nano-particle are subject to the same microscopic damping processes due to phonons, impurities, electronelectron interactions, etc., as off-resonance excitations. None of these inelastic scattering mechanisms is reduced or absent if the electrons oscillate near the resonance peak. The theoretical analysis revealed that the peculiar dependence of the effective radiation damping on laser frequency and depolarization factor leads to asymmetric spectral peaks and different lifetimes under on- or off-resonance conditions. Although other effects such as inter-particle and multipole interactions might affect the detailed shapes of the extinction and two-photon photo-emission spectra, the qualitative trend discussed here for radiation damping in ellipsoidal particles is expected to hold quite generally.

ACKNOWLEDGEMENTS One of the authors (A.L.) gratefully acknowledges the hospitality of the Donostia International Physics Center (San Sebastian, Spain), where part of this work was carried out, and stimulating discussions with A. Bringer. This work was supported by the Deutsche Forschungsgemeinschaft (DFG), the Austrian Ministry for Technology, the Austrian Science Foundation (grant P14292), and the European Union (TMR project NanoSNOM). 


\section{REFERENCES}

1 C. Bohren, D. Huffmann: Absorption and scattering of light by small particle (Wiley, New York 1983)

2 U. Kreibig, M. Vollmer: Optical properties of metal clusters (Springer, Berlin 1995)

3 N.W. Ashcroft, N.D. Mermin: Solid State Physics (Holt, Philadelphia 1976)

4 B. Lamprecht, A. Leitner, F.R. Aussenegg: Appl. Phys. B 68, 419 (1999)

5 F. Stietz, J. Bosbach, T. Wenzel, T. Vartanyan, A. Goldmann, F. Träger: Phys. Rev. Lett. 84, 5644 (2000); T. Vartanyan, M. Simon, F. Träger: Appl. Phys. B 68, 425 (1999)

6 J. Lehmann, M. Merschdorf, W. Pfeiffer, A. Thon, S. Voll, G. Gerber: Phys. Rev. Lett. 85, 2921 (2000)

7 T. Klar, M. Perner, S. Grosse, G. von Plessen, W. Spirkl, J. Feldmann: Phys. Rev. Lett. 80, 4249 (1998)

8 J.H. Klein-Wiele, P. Simon, H.G. Rubahn: Phys. Rev. Lett. 80, 45 (1998)

9 C.A. Schmuttenmaer, M. Aeschlimann, H.E. Elsayed-Ali, R.J.D. Miller, D. Mantell, J. Cao, Y. Gao: Phys. Rev. B 50, 8957 (1994)
10 W. Gotschy, K. Vonmetz, A. Leitner, F.R. Aussenegg: Appl. Phys. B 63, 381 (1996); Opt. Lett. 21, 1099 (1996)

11 M. Scharte, R. Porath, T. Ohms, M. Aeschlimann, J.R. Krenn, H. Ditlbacher, F.R. Aussenegg: to be published

12 M. Aeschlimann, M. Bauer, S. Pawlik: Chem. Phys. 205, 127 (1996)

13 A. Wokaun, J.P. Gordon, P.F. Liao: Phys. Rev. Lett. 48, 957 (1982)

14 J. Gersten, A. Nitzan: J. Chem. Phys. 75, 1141 (1981)

15 J.D. Jackson: Classical Electrodynamics (Wiley, New York 1975), Chap. 17; L.D. Landau, E.M. Lifshitz: The Classical Theory of Fields (Pergamon, Oxford 1965), Sect. 75

16 P.B. Johnson, R.W. Christy: Phys. Rev. B 6, 4370 (1972)

17 J. Tiggesbäumker, L. Köller, K.H. Meiwes-Broer, A. Liebsch: Phys. Rev. A 48, 1749 (1993)

18 J.J. Quinn: Phys. Rev. 126, 1453 (1962)

19 P.M. Echenique, J.N. Pitarke, E.V. Chulkov, A. Rubio: Chem. Phys. 251, 1 (2000)

20 R. Keyling, W.-D. Schöne, W. Ekardt: Phys. Rev. B 61, 1670 (2000)

21 M. Aeschlimann, B. Knorren, K.H. Bennemann: Appl. Phys. A 71, 485 (2000) 